\title{
A VARIATIONAL PROBLEM FOR SUBMANIFOLDS OF EUCLIDEAN SPACE
}

\author{
JOSEPH A. ERBACHER
}

\begin{abstract}
Let $M^{n}$ be a compact differentiable manifold and $R^{n+k}$ Euclidean space. A necessary and sufficient condition is given for an immersion $\psi: M^{n} \rightarrow R^{n+k}$ to be a stationary immersion for $J=\int . x_{y}^{n}\left\langle\boldsymbol{x}-\boldsymbol{x}_{c}, \boldsymbol{x}-\boldsymbol{x}_{c}\right\rangle d v$ subject to the side condition $V=$ $\int . u_{y}^{n} d v=$ a fixed constant, where $x_{c}$ is the center of mass. In particular, minimal submanifolds of spheres satisfy this condition.
\end{abstract}

1. Introduction. Let $M^{n}$ be an $n$-dimensional compact differentiable manifold. An immersion $\psi: M^{n} \rightarrow R^{n+k}$ induces a Riemannian metric on $M^{n} ; M^{n}$ with this Riemannian metric is denoted by $M_{\psi}^{n}$. Let $x$ denote the position vector in $R^{n+k}$, and let $\boldsymbol{x}_{c}$ denote the center of mass of $M_{\psi}^{n}$ in $R^{n+l}$; i.e., $\boldsymbol{x}_{c}=(1 / V) \int_{M_{\psi}^{n}} \boldsymbol{x} d v$, where $V=\int_{M M_{\psi}^{n}} d v$ and $d v$ is the volume element on $M_{\psi}^{n}$. For $p \in M_{\psi}^{n}$, the tangent space $T_{p}\left(M_{\psi}^{n}\right)$ is identified with a subspace of $T_{\psi(p)}\left(R^{n+k}\right)$. The normal space $T_{p}^{\frac{1}{p}}\left(M_{\psi}^{n}\right)$ is the subspace of $T_{\psi(p)}\left(R^{n+k}\right)$ consisting of all $X \in T_{\psi(p)}\left(R^{n+k}\right)$ which are orthogonal to $T_{p}\left(M_{\psi}^{n}\right)$. For $q \in R^{n+k}, T_{q}\left(R^{n+k}\right)$ is identified with $T_{0}\left(R^{n+k}\right)$ by parallel translation, where 0 is the origin in $R^{n+k}$; and $T_{0}\left(R^{n+k}\right)$ is identified with $R^{n+k}$. If $z: M^{n} \rightarrow R^{n+k}$, we consider $z$ as a vector field defined along $\psi$ by the above identifications. Let $z_{N}(p)$ be the orthogonal projection of $z(p)$ into $T_{p}^{\perp}\left(M_{\psi}^{n}\right)$ and $z_{T}$ the orthogonal projection of $z(p)$ into $T_{p}\left(M_{\psi}^{n}\right)$. The Euclidean inner product will be denoted by $\langle$,$\rangle .$

THEOREM. The immersion $\varphi: M^{n} \rightarrow R^{n+k}$ is a stationary immersion for $J=\int_{I_{\psi}^{n}}^{n}\left\langle\boldsymbol{x}-\boldsymbol{x}_{c}, \boldsymbol{x}-\boldsymbol{x}_{c}\right\rangle d v$ subject to the side condition $V=\int_{M I_{\psi}^{n}} d v=$ a fixed constant, say $V_{0}$, if and only if $\left(\boldsymbol{x}-\boldsymbol{x}_{c}\right)_{\Lambda}=\frac{1}{2}\left(\left\langle\boldsymbol{x}-\boldsymbol{x}_{c}, \boldsymbol{x}-\boldsymbol{x}_{\boldsymbol{c}}\right\rangle+\lambda\right) \eta$ and $\int_{M I_{\psi}^{n}} d v=V_{0}$, where $\lambda$ is a constant and $\eta$ is the mean curvature normal [2, p. 34]. ${ }^{1}$

The stationary character of $\varphi$ means that if $\psi_{t}, t \in(-\varepsilon, \varepsilon)$, is any one parameter family of immersions with $\psi_{0}=\varphi$ and $V_{0}=\int \Lambda_{\psi t}^{n} d v$ for all $t$, then $d J(0) / d t=0$.

Received by the editors July 23, 1971 and, in revised form, November 1, 1971.

AMS 1970 subject classifications. Primary 49F99, 53B25.

Key words and phrases. Compact differentiable manifold, Riemannian, Euclidean space, center of mass, stationary immersion, mean curvature normal, minimal submanifold.

${ }^{1}$ The definition of $\eta$ in [2] differs from our usage by a factor of $1 / n$. 
LeMma. The immersion $\varphi: M^{n} \rightarrow R^{n+k}$ satisfies

$$
x-x_{c}=\frac{1}{2}\left(\left\langle x-x_{c}, x-x_{c}\right\rangle+\lambda\right) \eta,
$$

$\lambda$ a constant, if and only if $\varphi$ immerses $M^{n}$ as a minimal submanifold of $a$ sphere $S^{n+k-1}$.

As an immediate consequence of the Theorem and the Lemma we have:

COROLlaRY. If $\varphi: M^{n} \rightarrow R^{n+k}$ immerses $M^{n}$ as a minimal submanifold of a sphere $S^{n+k-1}$ with $V_{0}=\int_{M_{\varphi}^{n}} d v$, then $\varphi$ is a stationary immersion for $J$ subject to $V=V_{0}$.

Brian Smyth has pointed out the following Proposition to me.

PROPOSITION. If $\varphi: M^{n} \rightarrow R^{n+k}$ is a stationary immersion for $J$ subject to $V=$ constant and in addition $\langle\eta, \eta\rangle$ is constant on $M^{n}$, then $\varphi$ immerses $M^{n}$ as a minimal submanifold of a sphere.

All immersions, vector fields, etc. are assumed to be $C^{\infty}$.

\section{Proofs.}

Proof OF THEOREM. Let $\psi_{t}$ be a 1-parameter family of immersions of $M^{n}$ into $R^{n+k}$ with $\psi_{0}=\varphi$. Let $y$ denote the position vector in $R^{n+k}$ for $\psi_{t}$ and $\boldsymbol{x}$ the position vector for $\varphi$. Assume $\boldsymbol{x}_{c}=\mathbf{0}$. Let $\boldsymbol{u}=d \boldsymbol{y}(0) / d t$. It is well known that (see $\left[1\right.$, p. 74 ] for the case of a surface in $R^{3}$ )

$$
\begin{aligned}
\frac{d V}{d t}(0) & =-\int_{M_{\varphi}^{n}}\langle\eta, \boldsymbol{u}\rangle d v+\int_{\boldsymbol{M}_{\varphi}^{n}} \operatorname{Div} \boldsymbol{u}_{T} d v \\
& =-\int_{M_{\varphi}^{n}}\langle\eta, \boldsymbol{u}\rangle d v .
\end{aligned}
$$

For $d J(0) / d t$ we have

$$
\begin{aligned}
\frac{d J}{d t}(0)= & \int_{M_{\varphi}^{n}}\langle\boldsymbol{x}, \boldsymbol{x}\rangle\left(\langle-\eta, \boldsymbol{u}\rangle+\operatorname{Div} \boldsymbol{u}_{T}\right) d v \\
& +\int_{M_{\varphi}^{n}}\left(\frac{d}{d t}\left\langle\boldsymbol{y}-\boldsymbol{y}_{c}, \boldsymbol{y}-\boldsymbol{y}_{c}\right\rangle\right)(0) d v .
\end{aligned}
$$

Using $\boldsymbol{x}_{c}=\mathbf{0}$ (and therefore $\int_{M_{\varphi}^{n}}\langle\boldsymbol{x}, \boldsymbol{b}\rangle d v=0$ for a constant vector $\boldsymbol{b}$ ) and

$$
\begin{aligned}
\langle\boldsymbol{x}, \boldsymbol{x}\rangle \operatorname{Div} \boldsymbol{u}_{T} & =\operatorname{Div}\langle\boldsymbol{x}, \boldsymbol{x}\rangle \boldsymbol{u}_{T}-\left\langle\boldsymbol{u}_{T}, \operatorname{grad}\langle\boldsymbol{x}, \boldsymbol{x}\rangle\right\rangle \\
& =\operatorname{Div}\langle\boldsymbol{x}, \boldsymbol{x}\rangle \boldsymbol{u}_{T}-2\left\langle\boldsymbol{u}_{T}, \boldsymbol{x}\right\rangle \\
& =\operatorname{Div}\langle\boldsymbol{x}, \boldsymbol{x}\rangle_{\boldsymbol{u}_{T}}-2\left\langle\boldsymbol{u}, \boldsymbol{x}_{T}\right\rangle,
\end{aligned}
$$


we easily find

$$
\begin{aligned}
\frac{d J}{d t}(0) & =\int_{M_{\varphi}^{n}}\left\langle 2 \boldsymbol{x}-2 \boldsymbol{x}_{T}-\langle\boldsymbol{x}, \boldsymbol{x}\rangle \eta, \boldsymbol{u}\right\rangle d v \\
& =\int_{M_{\varphi}^{n}}\left\langle 2 \boldsymbol{x}_{N}-\langle\boldsymbol{x}, \boldsymbol{x}\rangle \eta, \boldsymbol{u}\right\rangle d v
\end{aligned}
$$

Since $M^{n}$ is compact, $\eta$ is not identically zero. Appealing to the wellknown method of Euler-Lagrange multipliers for variational problems with side conditions, we conclude from (1) and (2) that a necessary and sufficient condition for $\varphi$ to be a stationary immersion for $J$ subject to $V=$ constant is that there exist a constant $\lambda$ (the Euler-Lagrange multiplier) such that $2 x_{N}-\langle\boldsymbol{x}, \boldsymbol{x}\rangle \eta=\lambda \eta$; i.e.,

$$
2 \boldsymbol{x}_{N}=(\langle\boldsymbol{x}, \boldsymbol{x}\rangle+\lambda) \eta
$$

Proof of Lemma. (i) Assume $\boldsymbol{x}_{c}=\mathbf{0}$. Suppose $\boldsymbol{x}=\frac{1}{2}(\langle\boldsymbol{x}, \boldsymbol{x}\rangle+\lambda) \eta$. Clearly $x_{N}=x$. Let $X$ be tangent to $M^{n}$. Then $X\langle x, x\rangle=2\langle X, x\rangle=0$, since $x_{N}=x$. Thus $\langle\boldsymbol{x}, \boldsymbol{x}\rangle$ is constant on $M^{n}$ and $\boldsymbol{x}=$ (constant) $\eta$. This implies that $\varphi$ immerses $M^{n}$ as a minimal submanifold of a sphere with center at the origin.

(ii) Suppose $\varphi$ immerses $M^{n}$ as a minimal submanifold of a sphere with center $a$. Since $\boldsymbol{x}-\boldsymbol{a}=\mu \eta$ for some constant $\mu$, it suffices to show that $\boldsymbol{x}_{c}=\boldsymbol{a}$. Let $f=\langle\boldsymbol{x}, \boldsymbol{b}\rangle$, where $\boldsymbol{b}$ is a constant vector. Then $\Delta f=\langle\eta, \boldsymbol{b}\rangle$, where $\Delta$ is the Laplacian on $M^{n}$ (see [2, p. 340]). Hence, $\int_{M_{\varphi}^{n}}\langle\eta, b\rangle d v=0$; and thus $\int_{M_{\varphi}^{n}} \eta d v=\mathbf{0}$. But $\boldsymbol{x}-\boldsymbol{a}=\mu \eta$. Thus $\int_{M_{\varphi}^{n}}(\boldsymbol{x}-\boldsymbol{a}) d v=\mathbf{0}$; i.e., $\boldsymbol{x}_{c}=\boldsymbol{a}$.

Proof of Proposition. Assume $\boldsymbol{x}_{c}=\mathbf{0}$, and let $H=\langle\eta, \eta\rangle$. Let $f=$ $\frac{1}{2}\langle\boldsymbol{x}, \boldsymbol{x}\rangle$. Then, it is not difficult to show that $\Delta f=n+\langle\boldsymbol{x}, \eta\rangle$, where $\Delta$ is the Laplacian on $M^{n}$. At a local maximum of $f$, we must have $x=x_{N}$ and $\Delta f \leqq 0$. Thus, $\langle\boldsymbol{x}, \eta\rangle \leqq-n$; and using (3) we obtain $f \leqq(-2 n / H)-\lambda$ at a local maximum of $f$. Similarly, at a local minimum of $f$ we have $f \geqq$ $(-2 n / H)-\lambda$. Thus, $f$ is constant on $M^{n}$ and $x=$ (constant) $\eta$. This implies that $\varphi$ immerses $M^{n}$ as a minimal submanifold of a sphere.

REMARK 1. It would be interesting to know whether or not all solutions of the variational problem considered in this paper are minimal submanifolds of spheres.

REMARK 2.

$$
\iint_{I_{\varphi}^{n} \times 1 I_{\varphi}^{n}}\left\langle\boldsymbol{x}_{1}-\boldsymbol{x}_{2}, \boldsymbol{x}_{1}-\boldsymbol{x}_{2}\right\rangle d v_{1} d v_{2}=2 V \int_{M I_{\varphi}^{n}}\left\langle\boldsymbol{x}-\boldsymbol{x}_{c}, \boldsymbol{x}-\boldsymbol{x}_{c}\right\rangle d v,
$$

where $\boldsymbol{x}_{i}$ is the position vector and $d v_{i}$ the volume element for the $i$ th factor of $M_{\varphi}^{n} \times M_{\varphi}^{n}$. 


\section{REFERENCES}

1. E. Hopf, Lectures on differential geometry in the large, Notes, Stanford University, Stanford, Calif.

2. S. Kobayashi and K. Nomizu, Foundations of differential geometry. Vol. II, Interscience Tracts in Pure and Appl. Math., no. 15, vol. II, Interscience, New York, 1969. MR 38 \#6501.

Department of Mathematics, University of Southern California, Los ANGeles, CALIFORNIA 90007 